CERN-TH/95-130

\title{
Invisible Events with Radiative Photons at LEP
}

\author{
G. Montagna ${ }^{a}$, O. Nicrosini ${ }^{b}$ 田,F. Piccinini ${ }^{c}$ \\ and L. Trentadue ${ }^{b}$ 目色 \\ ${ }^{a}$ INFN, Sezione di Pavia, Italy \\ ${ }^{b}$ CERN, TH Division, Geneva, Switzerland \\ ${ }^{c}$ INFN, Sezione di Pavia, Italy
}

\begin{abstract}
A study of the radiative neutrino counting reaction $e^{+} e^{-} \rightarrow \nu \bar{\nu} \gamma$ at LEP1 and LEP2 energies is presented. An approximate expression for the spectrum of the observed photon is derived within the framework of the $p_{t}$-dependent structure function approach. This is compared with an exact expression and found in agreement within the foreseen experimental accuracy. This model describing single-photon radiation can be applied to the more general case of initial-state single-photon emission accompanying invisible final-state events. Higher-order QED corrections due to undetected initial-state radiation are also included. The implementation in a Monte Carlo event generator is briefly described.

e-mail:

montagna@pv.infn.it, nicrosini@vxcern.cern.ch, piccinini@pv.infn.it, trenta@vxcern.cern.ch
\end{abstract}

CERN-TH/95-130

May 1995

\footnotetext{
${ }^{1}$ On leave from INFN, Sezione di Pavia, Italy.

${ }^{2}$ On leave from Dipartimento di Fisica, Università di Parma, Parma, Italy.

${ }^{3}$ INFN, Gruppo Collegato di Parma, Sezione di Milano, Milan, Italy.
} 


\section{Introduction}

The radiative neutrino-counting reaction and, more in general, the singlephoton reactions, provide a very useful tool for measuring the number of light neutrinos and eventually detecting new-physics signals at LEP [1].

The lowest-order spectrum for single-photon production within the Standard Model is known [2]. For energies around the $Z^{0}$ peak this exact calculation agrees within about $1 \%$ with two different approximations [3, 4].

Concerning higher-order QED corrections, two different calculations and the corresponding codes do exist [4, 5]. Both are based on the QED structure function approach [6]. In the first one [4], QED corrections are implemented on the tree-level spectrum in the so-called Point Interaction Approximation (PIA) and the corresponding program is a semi-analytical code. In the second one [5], $\mathcal{O}(\alpha)$ plus higher-order QED corrections are included for the $Z^{0}$-exchange only and the contribution of the $W$-exchange diagrams is added at the tree level. This formulation is implemented in a Monte Carlo generator of unweighted events. However, as is known, the treatment of the QED radiative corrections around the $Z^{0}$ peak can become inadequate as the energy increases. A proper treatment of the hard photonic radiation has to be provided.

The purpose of this work is twofold:

i) to improve the approximations existing in the literature for the study of the reaction at LEP1 energies, in order to get reliable results for radiative neutrino counting as well as for single-photon events at LEP2; to provide a new result on the treatment of the hard-photon spectrum;

ii) to establish a general strategy for developing a single-photon library, inclusive of all the standard and non-standard signals. This last point is motivated by the relevance of the experimental studies of single-photon radiation accompanying invisible events at LEP for the search of possible new-physics effects.

The outline of the paper is as follows. In Sect. 2 the exact and approximate calculations for the spectrum of the radiative neutrino counting reaction at lowest order in the Standard Model are reviewed. A quantitative study of the approximations at LEP2 versus LEP1 energies will be performed and an approximate expression for the photonic spectrum will be presented. In Sect. 3 it will be shown how to include in the formulation higher-order initialstate QED corrections, and our results will be compared with existing ones. Some single-photon distributions of experimental interest will be shown and commented in Sect. 4. Finally, conclusions and possible perspectives on the subject are given in Sect. 5 . 


\section{Lowest-Order Calculations}

The production of neutrino-antineutrino pairs and of a single photon is described within the Standard Model of the electroweak interactions by $s$ - and $t$-channel diagrams involving $Z^{0}$ and $W^{ \pm}$exchange [1]. $W$ contributions occur only for the production of electron neutrinos and they can be distinguished in diagrams with single- $W$ exchange and diagrams with the exchange of two $W$ 's, the latter involving the non-Abelian trilinear coupling $W W \gamma$. On the basis of the above diagrams, the differential cross section in the photon energy $E_{\gamma}$ and angle $\cos \vartheta_{\gamma}$ has been calculated exactly [2] and its expression contains the $Z^{0}$ and $W$ squared amplitudes and the $W-Z^{0}$ interference. What is important to stress about this calculation is that it is performed keeping a finite value for the mass of the $W$ boson and, therefore, it applies without restrictions on the energy range.

However, for the energies around the $Z^{0}$ peak, two approximations are known in the literature and they both reproduce, with an accuracy of the order of $1 \%$, radiative neutrino counting experiments at LEP1. The two approximations are:

- The Point Interaction (or Contact) Approximation [3] (PIA). It consists in neglecting the diagram with exchange of two $W$ 's and taking the limit $M_{W} \rightarrow \infty$ in the calculation.

- The Point Interaction Approximation with Angular Radiator [4] (PIAH). It uses the QED $p_{t}$-dependent structure functions, i.e. the method containing also the transverse degrees of freedom of the emitted radiation [7]. It consists in dressing the cross section for producing a $\nu \bar{\nu}$ pair (without photons) with an angle-dependent radiator. The photon spectrum is given in this approximation by the following factorized expression:

$$
\frac{d \sigma}{d x_{\gamma} d c_{\gamma}}=\sigma_{0}^{P I A}\left(\left(1-x_{\gamma}\right) s\right) H^{(\alpha)}\left(x_{\gamma}, c_{\gamma} ; s\right)
$$

$\sigma_{0}^{P I A}$ is the cross section for $e^{+} e^{-} \rightarrow \nu \bar{\nu}$ as evaluated within the PIA approximation at the energy scale reduced by photon emission; $H^{(\alpha)}\left(x_{\gamma}, c_{\gamma} ; s\right)$ is the angle-dependent radiator which describes the probability of radiating a photon with a given energy fraction $x_{\gamma}=E_{\gamma} / E_{b}$ at the angle $\vartheta_{\gamma}\left(c_{\gamma} \equiv \cos \vartheta_{\gamma}\right)$. Its expression, derived from the $\mathcal{O}(\alpha) p_{t}$-dependent structure function based on the electron $\rightarrow$ electron + photon splitting function, reads as [4]

$$
H^{(\alpha)}\left(x_{\gamma}, p_{t} ; s\right)=\frac{\alpha}{2 \pi} P\left(1-x_{\gamma}\right) \frac{1}{\pi}\left[\frac{1}{p_{t e^{+}}^{2}+x_{\gamma}^{2} m^{2}}+\frac{1}{p_{t e^{-}}^{2}+x_{\gamma}^{2} m^{2}}\right]+\mathcal{O}\left(\frac{p_{t}^{2}}{E_{\gamma}^{2}}\right),
$$


where $P(1-x)$ is the Altarelli-Parisi splitting function and $p_{t e^{-,+}}$represent the electron and positron transverse momenta, respectively. In terms of the photon angle $c_{\gamma}$, one obtains [4]

$$
H^{(\alpha)}\left(x_{\gamma}, c_{\gamma} ; s\right)=\frac{\alpha}{\pi} \frac{1+\left(1-x_{\gamma}\right)^{2}}{x_{\gamma}} \frac{1}{1+4 m^{2} / s-c_{\gamma}^{2}} .
$$

This expression contains the leading infrared and collinear singularities represented by the $1 / x_{\gamma}$ term and by the angular factor in (2), respectively. This specific expression can, however, be properly modified to include less dominant (next-to-leading) contributions, as will be shown in the following (see [四).

Figure 1 shows the integrated radiative neutrino counting cross section with the minimum photon tagging angle $\vartheta_{\gamma}^{\min }=20^{\circ}$ and the minimum photon energy $E_{\gamma}^{\min }=1 \mathrm{GeV}$. In this plot, the solid line is the result obtained by integrating the exact photon spectrum of Ref. [2], the dotted and dashed lines correspond to the PIA [3] and PIAH [4] approximations, respectively. As can be seen, the two approximations are in good agreement with the exact calculation around the $Z^{0}$ peak, but they start to differ as the energy goes above about $120-130 \mathrm{GeV}$ and the difference is well visible at higher LEP2 energies. Figure 2 is a blow-up of Fig. 1 at LEP1 (Fig. 2a) and LEP2 (Fig. 2b) energies respectively. The two approximations agree with the exact calculation within $\simeq 1 \%$ at LEP1 (where they essentially provide the same integrated cross section with a hard photon tail above the peak due to the emission of a $Z^{0}$ return photon of energy $\left.E_{\gamma} \simeq\left(1-M_{Z}^{2} / s\right) \sqrt{s} / 2\right)$, whereas at LEP2 they are in net disagreement with the full spectrum. In particular, they provide an increase in cross section with energy, as a consequence of the absence of finite $W$-mass effects needed at LEP2 energies. Furthermore, the larger deviation of the PIAH approximation is due, in particular, to the specific expression employed for the angular radiator as in eq. (2).

However, hard photons, strongly suppressed around the $Z^{0}$ peak, are important at LEP2 and they have to be included. Within the framework of the $p_{t}$-dependent structure function approach, by virtue of its generality, a simple approximate expression can be achieved, which constitutes one of the results of the present paper. This is of some importance for experiments at LEP2 because, if a proper expression for handling the photon spectrum is found, it can be used as a basic tool to develop a library of single-photon events, including standard and non-standard (in particular SUSY) processes. The approximate expression can be implemented with the two following steps:

- to replace the cross section $e^{+} e^{-} \rightarrow \nu \bar{\nu}$ in PIA approximation $\left(\sigma_{0}^{P I A}\right)$ with 
the corresponding exact cross section in the Standard Model $\left(\sigma_{0}^{\text {exact }}\right)$, i.e. for $e^{+} e^{-} \rightarrow(Z, W) \rightarrow \nu \bar{\nu}$, thus including the necessary $W$-mass effects $\rightarrow$;

- to adopt, instead of the form in eq. (2), the more accurate expression for the angular radiator in such a way that also a part of next-to-leading contributions are included. This properly modified radiator has been already derived [4] and reads:

$$
H_{S H}^{(\alpha)}=\frac{\alpha}{2 \pi} \frac{1}{x_{\gamma}}\left[2 \frac{1+\left(1-x_{\gamma}\right)^{2}}{1+4 m^{2} / s-c_{\gamma}^{2}}-x_{\gamma}^{2}\right] .
$$

The latter expression differs from the radiator of eq. (2) by less than $1 \%$ around the $Z^{0}$ peak, but it is more appropriate to describe the emission of hard photons (of energy fraction $x_{\gamma} \simeq 1$ ) at large angles.

Therefore the photonic spectrum of the reaction $e^{+} e^{-} \rightarrow \nu \bar{\nu} \gamma$ can be written in factorized form as follows:

$$
\frac{d \sigma}{d x_{\gamma} d c_{\gamma}}=\sigma_{0}^{\text {exact }}\left(\left(1-x_{\gamma}\right) s\right) H_{S H}^{(\alpha)}\left(x_{\gamma}, c_{\gamma} ; s\right) .
$$

Let us comment about the physical meaning of the above equation. Given the specific bare kernel $e^{+} e^{-} \rightarrow \nu \bar{\nu}$, as well as for any other kernel corresponding to any given process of the type $e^{+} e^{-} \rightarrow$ invisible objects, dressing it with the angular radiator $H_{S H}^{(\alpha)}$ amounts to attaching a photon line on the external charged legs; in this way, the "universal", factorized form of the photonic radiation will be included, comprehensive of some of the next-to-leading effects. Therefore, in this approximation the $W$ diagram with the photon emitted by the internal propagator is not reproduced.

However, by virtue of the generality of the method, if the cross section for an $e^{+} e^{-}$scattering yielding an invisible final state is known, an approximation of the cross section for the corresponding radiative process can be obtained in a straightforward way.

Figure 3 shows a comparison of the exact spectrum with the approximate results obtained using the two different angle-dependent radiators of (2) and (3), respectively. The solid line corresponds to the exact spectrum; the dotted and dashed lines are the result derived from convoluting the exact bare cross section with the angular radiators of eq. (2) and eq. (3), respectively.

As can be clearly seen, whereas the approximation based on the radiator of eq. (2) differs by several per cent from the exact result, using the "modified" radiator of eq. (3) improves the agreement at the level of a few per cent. To be more precise, for $150 \leq \sqrt{s} \leq 175$ the relative difference between the exact

\footnotetext{
${ }^{4}$ The exact invisible cross section was computed by means of SCHOONSCHIP \&.
} 
results and the approximation of eq. (4) is of the order of $1-2 \%$ and becomes of the order of 3-4\% only at about $200 \mathrm{GeV}$. These deviations, depending on the centre-of-mass energy, can be seen as a measure of the non-leading effects not included in the angular radiator approximation. However, for the experimental precision foreseen at LEP2, a theoretical error of the order of a few per cent is more than adequate.

\section{Higher-Order QED Corrections}

To match the experimental precision expected at LEP2, the corrections due to multiphoton soft emission and to the radiation of hard photons emitted in the very forward direction (and hence lost in the beam pipe) by the colliding beams must be included. The large QED corrections introduced by the undetected initial-state radiation can be successfully described within the framework of the QED structure function approach. This method, as recalled in the Introduction, is indeed employed in both the formulations developed for radiative neutrino counting at LEP1 [1, 5]. However, both the approaches have their drawback and therefore need to be updated for experiments at LEP2. A simple solution, which represents an improvement of both the existing approaches and has an overall accuracy of about $1 \%$, can be achieved by convoluting the exact spectrum of Ref. [2] with electron and positron structure functions. The QED-corrected cross section can then be written, in analogy with QCD factorization, as a convolution of the following form [9]:

$$
\sigma(s)=\int d x_{1} d x_{2} d E_{\gamma} d c_{\gamma} D\left(x_{1}, s\right) D\left(x_{2}, s\right) \frac{d \sigma}{d E_{\gamma} d c_{\gamma}},
$$

where $d \sigma / d E_{\gamma} d c_{\gamma}$ is the exact spectrum of $e^{+} e^{-} \rightarrow \nu \bar{\nu} \gamma$ [2], the photon variables being referred to the centre-of-mass frame after initial-state radiation, and $D(x, s)$ is the electron (positron) structure function. Its expression, as obtained by solving the Lipatov-Altarelli-Parisi evolution equation in the nonsinglet approximation, is given by [6]:

$$
\begin{aligned}
D(x, s) & =\frac{\exp \left\{\frac{1}{2} \beta\left(\frac{3}{4}-\gamma_{E}\right)\right\}}{\Gamma\left(1+\frac{1}{2} \beta\right)} \frac{\beta}{2}(1-x)^{\frac{\beta}{2}-1}-\frac{\beta}{4}(1+x) \\
& +\frac{1}{32} \beta^{2}\left[-4(1+x) \ln (1-x)+3(1+x) \ln x-4 \frac{\ln x}{1-x}-5-x\right],
\end{aligned}
$$

with

$$
\beta=2 \frac{\alpha}{\pi}(L-1)
$$


where $L=\ln \left(s / m^{2}\right)$ is the collinear logarithm, $\gamma_{E}$ is the Euler constant and $\Gamma(z)$ is the gamma function. The first exponentiated Gribov-Lipatov term describes multiphoton soft emission, the second and third ones hard bremsstrahlung in the collinear approximation. Further non-leading QED corrections are included as a $K$-factor, computed from the exact $O(\alpha)$ result of Ref. [10]. The relevant weak corrections are taken into account, for the $Z^{0}$-exchange contribution, in the form of Improved Born Approximation, according to the general recipe of Ref. [11].

The master formula (5) is implemented in a Monte Carlo event generator 13 . F. Figure $4 \mathrm{a}$ shows the QED-corrected cross section as a function of the centre-of-mass energy at LEP2. The dash-dotted line represents the exact cross section at lowest order; the solid line is the result corresponding to eq. (5) (i.e. in the case of convolution of the full spectrum), and the dotted line, reported for the sake of comparison, shows the results obtained by simulating the approach of Ref. [5], namely correcting the $Z^{0}$ contribution only, and adding to this result the $W$-exchange diagrams at tree level. Two considerations are in order here. First, the QED-corrected cross section is higher than the Born one as a consequence of the $Z^{0}$ radiative return: the effect is to enhance the Born result by a factor of about 1.3. Secondly, the convolution of the full spectrum is in good agreement (within 1\%) with the approach of Ref. [5] because the QED-corrected cross section is largely dominated by the $Z^{0}$ radiative return, and the tree-level contribution of $W$ diagrams (and $W-Z$ interference) is almost flat over the full energy range spanning from LEP1 to LEP2 (Fig. 4b). Of course, a more general (beyond the Standard Model) single-photon spectrum, including initial-state QED corrections, can be simply obtained. This can be achieved by substituting, in eq. (5), the exact proper generalized spectrum (or, if sufficient, its approximation according to eq. (4)) for $d \sigma / d E_{\gamma} d c_{\gamma}$ within the Standard Model.

\section{Simulation of Photon Distributions}

In this section, some technical details about the Monte Carlo event generator developed on the basis of the formulation presented in the previous section will be sketched; a complete and exhaustive account of the code will be given elsewhere [13]. A sample of realistic experimental distributions obtained by analysing the single-photon events generated by the Monte Carlo code will also be shown and briefly discussed.

\footnotetext{
${ }^{5}$ For a first attempt to develop a neutrino counting Monte Carlo based on $p_{t}$-dependent structure functions, see Ref. [12.
} 
Since the integrand is a superposition of different peaking behaviours, the standard importance-sampling technique, within the multichannel approach, was employed to develop an efficient event generator. The QED-corrected cross section is written as the sum of four different channels with the following features: (1) the radiative variables $x_{1,2}$ are generated according to the soft exponentiated part of the structure function, and the photon energy is then generated according to the dependence of the $Z^{0}$ propagator on $E_{\gamma}$; (2) $x_{1,2}$ as above and $E_{\gamma}$ generated according to the infrared-like behaviour $1 / E_{\gamma}$; (3) $E_{\gamma}$ flat, $x_{1}$ as the soft part of the structure function and $x_{2}$ according to the dependence of the $Z^{0}$ propagator on the reduced centre-of-mass energy $\hat{s}=x_{1} x_{2} s$; (4) the same as (3) with the interchange of $x_{1} \leftrightarrow x_{2}$. Moreover, to take care of the Lorentz boost caused by the emission of unbalanced electron and positron radiation, the photon phase-space variables are first generated in the centre-of-mass frame and then boosted to the laboratory frame by the boost given by the Lorentz factor $\beta_{L}=\left(x_{1}-x_{2}\right) /\left(x_{1}+x_{2}\right)$ valid for collinear kinematics.

Figures 5, 6 and 7 show the photon energy distribution, the photon angular distribution and the $p_{t}^{\gamma} / E_{b}$ distribution at a fixed LEP1 $\left(E_{b}=48 \mathrm{GeV}\right)$ and LEP2 $\left(E_{b}=88 \mathrm{GeV}\right)$ beam energies, respectively, assuming an experimental apparatus with $E_{\gamma}=1 \mathrm{GeV}$ and $\vartheta_{\gamma}^{\min }=20^{\circ}$. The solid histograms correspond to the lowest-order approximation, the dashed ones include the effect of initial-state radiation. In order to consistently compare the tree-level and QED-corrected distributions, the numbers of events integrated in the two cases are proportional to the corresponding integrated cross sections. Concerning the photon energy distribution (Fig. 5), two peaks are well visible both at LEP1 (Fig. 5a) and LEP2 (Fig. 5b) energies: the higher one is located at the energy value of about $\left(1-M_{Z}^{2} / s\right) \sqrt{s} / 2$, the lower one is due to $1 / E_{\gamma}$ peaking behaviour. As can be seen, the main modifications introduced by initial-state radiation are to reduce the higher peak and to enhance the lower one, as expected by typical convolution effects, thus modifying the two-bump asymmetrical shape of the Born distribution into a more symmetrical one. The photon angular distribution (Fig. 6) has the same symmetrical shape (peaked in the forward direction) at LEP1 (Fig. 6a) and LEP2 (Fig. 6b) energies, the only difference being that at LEP2 the QED-corrected distribution is higher than the Born one because of the $Z^{0}$ radiative return. At last, comparing the $p_{t}^{\gamma} / E_{b}$ distributions at LEP1 (Fig. 7a) and LEP2 (Fig. 7b) energies the emission of mostly soft photons at LEP1 and of harder ones at larger angles at LEP2 can be clearly recognized. 


\section{Conclusions}

In this paper a study of the neutrino counting reaction $e^{+} e^{-} \rightarrow \nu \bar{\nu} \gamma$ at LEP2 energies has been presented. Within the $p_{t}$-dependent structure function approach, an approximate expression for the photon energy and angle spectrum has been derived. The accuracy of the approximation, as estimated by comparison with the exact result, is of the order of $1-2 \%$ near the $W$-pair threshold and grows larger (up to about 3-4\%) only at about $200 \mathrm{GeV}$. By virtue of its generality, the approximation can be used as a first attack strategy to dress standard as well as non-standard (e.g. SUSY) processes giving rise to an invisible final state in order to get an approximate formula for the spectrum of the corresponding radiative single-photon events. Therefore, on the basis of these considerations, a library of single-photon processes should be feasible for experiments at LEP2. A further investigation on the tree-level generalized spectra would be of course required for processes whose form is less simple than the one shown in eq. (4).

Concerning higher-order QED corrections, a formulation based on structure function convolution of the exact photon spectrum is presented, which can be estimated to be accurate at the $1 \%$ level and agrees with the approximate

approach of Ref. [5] within about 1\%. This is implemented in a Monte Carlo event generator 13], which also can be easily generalized to describe singlephoton radiative processes accompanying invisible events at LEP.

\section{Acknowledgements}

Useful discussions with B. Mele and G. W. Wilson are gratefully acknowledged.

\section{References}

[1] L. Trentadue et al., Neutrino Counting, in Z Physics at LEP1, G. Altarelli, R. Kleiss and C. Verzegnassi, eds., CERN 89-08, Vol. 1 (1989) p. 129, and references therein.

[2] F. A. Berends et al., Nucl. Phys. B301 (1988) 583.

[3] K. J. F. Gaemers, R. Gastmans and F. M. Renard, Phys. Rev. D19 (1979) 1605 ;

G. Barbiellini, B. Richter and J. L. Siegrist, Phys. Lett. B106 (1981) 414.

[4] O. Nicrosini and L. Trentadue, Nucl. Phys. B318 (1989) 1. 
[5] R. Miquel, C. Mana and M. Martinez, Z. Phys. C48 (1990) 309; also in QED Structure Functions, G. Bonvicini, ed., AIP Conf. Proc. No. 201 (AIP, New York, 1990), p. 395.

[6] E. A. Kuraev and V. S. Fadin, Sov. J. Nucl. Phys. 41 (1985) 466;

G. Altarelli and G. Martinelli, Physics at LEP, CERN Report 86-02, J. Ellis and R. Peccei, eds. (Geneva, 1986); see also:

O. Nicrosini and L. Trentadue, Phys. Lett. B196 (1987) 551; Z. Phys. C39 (1988) 479.

For a review see also:

O. Nicrosini and L. Trentadue, in Radiative Corrections for $e^{+} e^{-}$Collisions, J. H. Kühn, ed. (Springer, Berlin, 1989), p. 25; in QED Structure Functions, G. Bonvicini, ed., AIP Conf. Proc. No. 201 (AIP, New York, 1990), p. 12; O. Nicrosini, ibid., p. 73.

[7] O. Nicrosini and L. Trentadue, Phys. Lett. B231 (1989) 487, and references therein.

For a review see also:

O. Nicrosini and L. Trentadue, in Radiative Corrections for $e^{+} e^{-}$Collisions, J. H. Kühn, ed. (Springer, Berlin, 1989) p. 25; in QED Structure Functions, G. Bonvicini, ed., AIP Conf. Proc. No. 201 (AIP, New York, 1990), p. 12; O. Nicrosini, ibid., p. 73.

[8] SCHOONSCHIP, A Program for Symbol Handling by M. Veltman, see H. Strubbe, Comput. Phys. Commun. 8 (1974) 1.

[9] G. Montagna, O. Nicrosini and F. Piccinini, Phys. Rev. D48 (1993) 1021, and references therein;

M. Cacciari et al., Phys. Lett. B268 (1991) 441.

[10] M. Igarashi and N. Nakazawa, Nucl. Phys. B288 (1987) 301; H. Veltman, Nucl. Phys. B312 (1989) 1.

[11] M. Consoli, W. Hollik and F. Jegerlehner, Electroweak radiative corrections for $Z$ physics, in Z Physics at LEP1, G. Altarelli, R. Kleiss and C. Verzegnassi, eds., CERN 89-08, Vol. 1 (1989), p. 7, and references therein.

[12] G. Bonvicini and L. Trentadue, Nucl. Phys. B323 (1989) 253; also in QED Structure Functions, G. Bonvicini, ed., AIP Conf. Proc. No. 201 (AIP, New York, 1990), p. 216.

[13] G. Montagna, O. Nicrosini and F. Piccinini, in preparation (to be submitted to Comput. Phys. Commun.). 


\section{Figure Captions}

Figure 1. The lowest-order integrated cross section of $e^{+} e^{-} \rightarrow \nu \bar{\nu} \gamma$ for $E_{\gamma}=$ $1 \mathrm{GeV}$ and $\vartheta_{\gamma}^{\min }=20^{\circ}$ as a function of the centre-of-mass energy. Solid line: exact spectrum; dotted line: PIA; dashed line: PIA with angular radiator.

Figure 2. Blow-up of Fig. 1 at LEP1 (Fig. 2a) and LEP2 (Fig. 2b) energies.

Figure 3. The lowest-order integrated cross section at LEP2. Solid line: exact spectrum; dotted line: "soft" angular radiator of eq. (2); dashed line: "modified" angular radiator of eq. (3).

Figure 4. The QED-corrected integrated cross section at LEP2 (Fig. 4a). Solid line: convolution of the exact spectrum; dotted line: convolution of the $Z^{0}$ contribution with $W$ at tree level; dash-dotted line: exact Born spectrum. The $W$ and $W-Z^{0}$ interference contributions as a function of the centre-ofmass energy (Fig. 4b).

Figure 5. The photon energy distributions at LEP1 (Fig. 5a) and LEP2 (Fig. 5b) energies. Solid histogram: Born; dashed histogram: QED-corrected.

Figure 6. The same as Fig. 5 for the photon angular distribution.

Figure 7. The same as Fig. 5 for the $p_{t}^{\gamma} / E_{b}$ distribution. 